\title{
ДИНАМИКА ПОЗНАВАТЕЛЬНЫХ ДЕЙСТВИЙ: AGM и DDL
}

\section{Н.П. Козаченко}

Раздел современной эпистемологии, который занимается изучением процесса изменения знаний и убеждений (belief revision), охватывает довольно обширную сферу исследований. В рамках ревизии убеждений рассматривается онтологический статус знания и убеждения, связь между ними, способы приобретения знаний и убеждений, возможности формализации человеческого знания, возможные области применения построенных формальных систем. Наиболее активно развиваются направления исследований, связанные с анализом самого процесса изменения знаний. В рамках такого рода анализа, основное внимание исследователей сосредоточено на выражении непосредственно динамики изменения убеждений. Существуют две основные концепции, на основе которых разрабатываются и уточняются все новые системы изменения убеждений, в рамках которых исследователи пытаются найти оптимальный способ отразить динамику изменения убеждений. Основные концептуальные разработки в этой сфере инициированы во второй половине XX века и в дальнейшем осуществлены в рамках так называемой теории AGM. Свое оригинальное название теория получила по первым буквам в фамилиях ее создателей Карлоса Алчуррона, Питера Герденфорса, Дэвида Макинсона, которые изложили основные идеи концепции в совместной публикации [1]. Подход, разрабатываемый в рамках концепции AGM, предполагает детальное изучение механизмов изменения убеждений и познавательных действий как функций над множествами убеждений (теориями). Более «молодая» стратегия DDL - динамическая доксастическая логика, предложенная Кристером Сегербергом [10], послужила основанием для развития 
многочисленных динамических логик убеждений, которые используют тщательно проработанный и выразительный язык DDL. Несмотря на очевидные преимущества DDL в некоторых аспектах анализа изменения убеждений, в рамках концепции AGM осуществляется не меньшее количество исследований динамики убеждений. В чем же основные отличия указанньх подходов, исключают ли они друг друга или дополняют, возможно ли хотя бы частичное их объединение? Для ответа на эти и другие вопросы относительно соотношения AGM и DDL кратко рассмотрим основные принципы этих подходов.

\section{1 Концепция AGM}

Ставшая своего рода классикой изменения убеждений, теория AGM подразумевает функциональный подход к анализу познавательных действий (см., напр.: [2], [3]). Рассматриваются три основные познавательные операции.

Операция расширения - простейший оператор изменения убеждений в AGM. Суть его состоит в добавлении новой информации к исходному множеству убеждений, теории. Расширение реализуется с помощью функции + , которая отображает пару (множество убеждений, высказывание) на множество множеств убеждений. Расширение множества убеждений характеризует ряд постулатов.

Ex1 Замыкание: Множество убеждений, полученное в результате расширения исходного путем добавления высказывания $\alpha$, также должно быть множеством убеждений, т. е. замкнутым относительно логического следования и непротиворечивым.

Ex2 Успешность: Расширение множества убеждений за счет высказывания $\alpha$ должно гарантировать присутствие $\alpha$ в результирующем множестве убеждений.

Ex3 Включение: Исходное множество убеждений должно быть подмножеством результирующего, полученного путем добавления некоторого высказывания к исходному множеству убеждений.

Ex4 Пустота: Если некоторое высказывание содержится во мнокестве убеждений, то расширение множества за счет этого высказывания никак не изменит исходное множество убеждений. 
Ex5 Монотонность: Расширение монотонно относительно операции включения. Результат расширения некоторого подмножества исходного множества убеждений за счет высказывания $\alpha$ также будет подмножеством результата расширения исходного множества посредством $\alpha$.

Рассмотренные постулаты позволяют описать семейство операторов расширения. Кроме того, для более полной характеристики операторов расширения следует указать, что исходная теория не должна быть расширена больше, чем этого требует добавление некоторой новой информации. Это требование явно отражено в постулате минимальности.

Ex6 Минимальность: Каким бы ни было множество убеждений, результат его расширения посредством любого высказывания должен быть наименьшим множеством убеждений, для которого выполняются постулаты замыкания, успешности и включения.

Результат расширения AGM может быть однозначно определен как множество убеждений, полученное путем теоретико-множественного объединения исходного множества убеждений и добавляемого высказывания и замкнутое относительно логического следования. Это подтверждает интуитивно приемлемую простоту оператора расширения.

Фактически расширение предполагает два шага:

1. Добавление высказывания к множеству убеждений путем теоретико-множественного объединения.

2. Замыкание полученного множества относительно операции логического следования.

Oперация сокращения используется в случае, когда некоторое высказывание подлежит удалению из множества убеждений без добавления какой-либо новой информации. Сокращение реализуется с помощью функции - , которая отображает пару (множество убеждений, высказывание) на множество множеств убеждений. Сокращение множества убеждений характеризует ряд постулатов.

C1 Замыкание: Множество убеждений, полученное в результате сокращения некоторой теории путем удаления высказывания $\alpha$, также должно быть множеством убеждений, т. е. замкнутым относительно логического следования и непротиворечивым. 
C2 Успешность: Если высказывание $\alpha$, подлежащее удалению, не является тавтологией, то сокращение множества убеждений за счет этого высказывания должно гарантировать отсутствие $\alpha$ в результирующем множестве убеждений.

C3 Включение: Исходное множество убеждений должно включать результат своего сокращения посредством любого высказывания.

C4 Пустота: Граничная ситуация, когда удаляемое высказывание не присутствует в исходном множестве и в результате применения оператора сокращения никаких изменений не произойдет.

C5 Экстенсиональность: Множества убеждений, полученные в результате сокращения некоторой теории посредством удаления высказываний, эквивалентных между собой, также будут эквивалентны.

Критерий экономии информации требует чтобы результат сокращения был максимальным подмножеством исходной теории, т.е. чтобы оператор сокращения полностью удалил только лишь высказывание, подлежащее удалению и ничего более. В качестве критерия минимальности сокращения предложен постулат восстановления.

C6 Восстановление: Расширение теории посредством добавления ранее удаленного высказывания должно полностью восстановить исходное множество убеждений в том виде, каким оно было до удаления этого высказывания.

Перечисленные выше постулаты являются основными AGM постулатами сокращения (или постулатами Герденфорса [2]). В дополнение к ним авторы AGM рассматривают постулаты, отражающие свойства сокращения посредством конъюнкции.

Операция ревизии (пересмотра) связана с расширением, т. к. предполагает введение новой информации в множество убеждений, но в то же время на нее возложен контроль непротиворечивости входящей информации и исходного множества. Поэтому функции ревизии, кроме простого введения новой информации, должны отследить возникновение противоречия и при необходимости избавиться от него. Таким образом, ревизия должна по мере надобности удалять некоторые высказывания, вступающие в противоречие с входящей информацией. Как и операцию сокращения, ревизию довольно тяжело определить 
однозначно, но возможно сформулировать набор постулатов, которым должно удовлетворять семейство функций ревизии. Ревизия реализуется с помощью функции *, которая отображает пару (множество убеждений, высказывание) на множество множеств убеждений. Ревизию множества убеждений характеризует следующее множество постулатов.

R1 Замыкание: Множество убеждений, полученное в результате ревизии исходной теории посредством высказывания $\alpha$, также должно быть множеством убеждений.

R2 Успешность: Согласно принципу приоритетности новой информации, высказывание, посредством которого производится ревизия, должно быть введено в теорию.

R3 Включение: Результат ревизии множества убеждений посредством высказывания $\alpha$ состоит из следствий высказывания $\alpha$ и подмножеств исходного множества убеждений, которые не противоречат $\alpha$.

R4 Пустота: Если исходная теория содержит отрицание высказывания, посредством которого производится ревизия, то простое добавление такого высказывания даст нам противоречивое множество убеждений. В случае, когда отрицание высказывания не содержится в исходной теории, т. е новая информация не противоречит никакому высказыванию исходного множества, то нет резона удалять что-либо из исходного множества.

R5 Непротиворечивость: Если новая информация внутренне непротиворечива, то результат ревизии также должен быть непротиворечивым.

R6 Экстенсиональность: Множества убеждений, полученные в результате пересмотра некоторой теории посредством эквивалентных высказываний, эквивалентны между собой.

Подход AGM довольно часто подвергают критике. В основном по двум направлениям - статичность и однозначность функционального представления операторов изменения. Познавательные операции AGM «пропускают» момент изменения убеждений, сопоставляя исходному 
множеству результирующее множество убеждений, полученное вследствие некоторого изменения исходного. Кроме того, это сопоставление представлено в виде функции, т. е. обеспечивает однозначность результата, не рассматривая возможные сопутствующие изменения в самом множестве убеждений. Также в рамках подхода AGM неотъемлемой частью анализа убеждений есть использование метаязыка, что не дает возможности полностью формализовать эту концепцию.

\section{2 Динамическая доксастическая логика DDL}

В работах Кристера Сегерберга подход AGM был подвергнут критике и предложено другое направление в исследованиях ревизии знаний и убеждений [10], [11]. Сегерберг указывает на то, что AGM не реальная логика, а скорее сорт некоторой математической метатеории о теориях. Это касается в первую очередь языка, в котором элементарные выражения есть скорее метавыражениями об объектах некоторой теории. Соответственно, вышеупомянутые типы изменения множеств убеждений рассматриваются в AGM как функции над теориями, что не дает возможности проследить динамику процесса изменения убеждений. В свою очередь, Сегерберг в рамках DDL вводит синтаксис, отличный от языка AGM. Язык DDL более гибок и выразителен и дает возможность более тщательно исследовать динамику изменения убеждений и знаний. В отличие от AGM, где познавательные действия реализованы как универсальные действия, независимые относительно выбора субъекта и высказывания, посредством которого осуществляется изменение, Сегерберг предлагает рассмотреть расширение, сокращение и ревизию не просто как функции, а как определённого рода действия, а именно как эпистемические или доксастические действия познающего субъекта. Он определяет познавательные действия как термообразующие операторы, которые преобразовывают булевы формулы в так называемые доксастические термы. С помощью такой терминологии он интерпретирует доксастические действия субъекта, ассоциируя их с высказываниями, посредством которых реализуется действие.

$+\psi$ - расширение посредством $\psi$,

* $\psi$ - ревизия посредством $\psi$,

$\div \psi$ - сокращение посредством $\psi$.

Следуюшие важные операторы теории DDL это скобки [] и $<>$, которые используются для построения динамических формул. Обозначим посредством $\phi$ некоторый доксастический терм $+\psi, * \psi$ или $\div \psi$. 
Тогда $[\phi] \varphi$ будет обозначать «после выполнения $\phi$ обязательно получим $\varphi »$. Аналогично $\langle\phi\rangle \varphi$ означает «после выполнения $\phi$ возможно получим $\varphi$ в теории». Скобки взаимовыразимы $-<\phi>\chi=\neg[\phi] \neg \chi$. Путем введения скобок K. Сегерберг указывает на то, что познавательные действия не порождают функциональную зависимость, в силу того, что могут не обеспечить однозначный результат. Синтаксис явно указывает индетерминированность доксастических операторов DDL. Результат выполнения одной и той же операции может быть разным. B рамках AGM имели бы место следующие эквивалентности: $<+\alpha>\chi \leftrightarrow[+\alpha] \chi$ и $<* \alpha>\chi \leftrightarrow[* \alpha] \chi$. Фактически, познавательные действия в AGM определены достаточно четко, чтобы субъект мог быть уверен в том, что выполняя, к примеру, расширение своего множества убеждений посредством одного и того же высказывания, он получит одинаковый результат. В DDL предвосхищается внутренняя активность, интроспективность самого субъекта, который оперирует своим множеством убеждений не только под влиянием внешних входов, а и по внутренней необходимости. Также в DDL синтаксисе рассматриваются два модальных доксастических оператора, K - знания и В - убежденности. Общий вид правильно построенной формулы в DDL может быть представлен формулой вида $[O \alpha] B \beta$, где $\mathrm{O}-$ некоторое доксастическое действие, В $(\mathrm{K})$ - оператор убежденности (знания), $\alpha$ и $\beta$ булевы.

Исследования, в основе которых лежит такая стратегия ревизии знаний, получили название Динамической Доксастической Логики (DDL). Основная идея DDL заключается в попытке представить теорию изменения как реальную логику, сходную с модальной, а также проследить саму динамику изменения теорий, в отличие от AGM, coсредоточенной на содержании познавательных операций. Для систем DDL и более поздних исследований характерно построение целостной формально-логической системы и полное рассмотрение ее свойств.

Направление исследований, иницированное в свое время DDL, порождает ряд ответвлений. В центре рассмотрения находятся не только динамика и индетерминизация доксастических действий, а и возможные типы субъектов, осуществляющих әти действия. Субъект может воспринимать внешнюю информацию, но может и не воспринимать, в зависимости от задачи, поставленной перед субъектом. Кроме того, доксастические действия субъекта могут быть инициированы и внутренней необходимостью. Безусловно, такая необходимость может возникнуть не у каждого субъекта - носителя множества убеждений. Наличие внутренних стимулов изменения убеждений порождает не- 
обходимость рассмотрения других доксастических операторов, отличных от расширения, сокращения и ревизии, а также специфические свойства традиционных операторов.

Условно можно выделить такие типы познающих субъектов [14]:

Неинтроспективный статический субъект. Например, фиксированная база данных, которая не зависит от изменений внешнего мира. В такой базе данных не предусмотрен вход извне, нет механизма для его обработки, да и надобности в такой обработки тоже нет. Проще всего представить промьшленого робота, который выполняет достаточно сложную, порой ювелирную, но четко определенную схему действий. Өто своего рода аналог доксастической логики Я. Хинтикки [6], в которой нет доксастических операторов для обработки полученной информации.

Интроспективный статический субъект. Доксастическая и эпистемическая логика Хинтикки, в которой субъект не обрабатывает внешнюю информацию, но по собственной инициативе производит внутренне ориентированные доксастические действия.

Неинтроспективный динамический субъект. Субъект, действующий аналогично динамической базе данных, воспринимающий внешнюю информацию, обрабатывающий ее и обучающийся в процессе приобретения опыта. Такой познающий субъект характерен для большинства теорий, разрабатываемых в рамках AGM подхода. Для основной концепции DDL, выразительная способность которой ограничена неитерируемостью доксастических операторов, интроспективность субъекта также не может быть реализована. В первичной системе DDL правильно построенными формулами есть формулы вида $[O \alpha] B \beta$, где - некоторое доксастическое действие, - оператор убежденности, при этом $\alpha$ и $\beta$ могут быть только булевыми.

Интроспективный динамический субъект, внешний доксастический вход которого ограничен высказываниями о внешнем мире. Характерен для расширенной концепции Extended DDL [8], для которой правильно построенными формулами есть формулы вида $[O \alpha] B \beta$, где $\mathrm{O}$ - некоторое доксастическое действие, В - оператор убежденности, при этом $\alpha$ может быть только булевым, а $\beta$ любым высказыванием. 
Интроспективный динамический субъект, доксастический вход которого может включать высказывания о его собственном эпистемическом состоянии. Реализован в полной системе DDL, так называемой Full DDL [8], [9]. В правильно построенной формуле вида $[O \alpha] B \beta$, где $O$ - некоторое доксастическое действие, $B$-оператор убежденности, $\alpha$ и $\beta$ могут быть любыми высказываниями, как о внешнем мире, так и о эпистемическом состоянии самого субъекта.

В полной системе DDL субъект может оперировать информационными входами любого характера. Высказывания, изменяющие множество убеждений субъекта, могут описывать как внешний мир, так и внутренние желания самого субъекта. Познавательные действия могут быть применены повторно, к результату своего предыдущего применения либо к другому познавательному действию, что невозможно для классических систем AGM.

Кроме интроспективного рассмотрения субъекта-носителя убеждений, исследователи обращают внимание и на взаимосвязь познавательных действий и общения. Субъект изменяет свое множество убеждений не только в результате процесса познания, производимого им самостоятельно, не только из собственных наблюдений, но и в результате взаимодействия с другими субъектами. Мнение, носителем которого есть не один субъект, а группа (common beliefs) [17], также представляет собой интересный объект для рассмотрения в рамках эпистемической логики. Исследования, которые опираются на логические системы DDL и подобные, активно разрабатывают субъективные, психологические и социальные аспекты динамики убеждений.

\section{3 Методология DEL}

Несмотря на заявленную динамичность, в рамках DDL представление процесса изменения убеждений осталось таким же статичным, как и в AGM. Но идея построения динамической логики не угасла, тем более DDL синтаксис предоставляет огромные выразительные возможности. На основе подхода, впервые рассмотренного Сегербергом, вскоре была разработана методология динамизации логик, с помощью которой можно построить различные динамические эпистемические логики (DEL) на основе уже разработанных формализованных систем [19]. Метод построения динамических эпистемических логик DEL основывается на результатах, полученных при рассмотрении ло- 
гик DDL синтаксиса и принципах их построения. Основными преимуществами DDL в качестве основы для создания расширенных аксиоматических систем можно назвать как минимум следующие:

- гибкий синтаксис;

- разработанная семантика;

- традиционный әкспансивный способ построения логических систем-надстроек: DDL - Extended DDL - Full DDL;

- наличие доказанных теорем о разрешимости и полноте аксиоматических систем DDL.

Основные принципы методологии DEL отражают широкий спектр ее применения.

Мультисубъектность эпистемических логик и соответственных моделей, пожалуй стоит назвать основной характеристикой методологии DEL. Рассматривается изменение убеждений не только единичного носителя убеждений, но и группы, взаимосвязанных, взаимодействующих субъектов, реагирующих на изменяющийся мир общения, информации, социума. Также стоит отметить стремление учесть современные психологические, социальные, игровые тенденции, исследования в области искусственного интеллекта, с целью объединить их и выразить в формально-логических системах.

Исследование причин изменения. Каждое изменение убеждений имеет «спусковой механизм» (trigger) [13]. Таким механизмом, порождающим изменения, может быть единичная констатация факта, общественное мнение, публичное заявление, глобальное информационное событие, взаимодействие субъектов, собственная активность субъекта [18]. Исходя из этого, минимальный набор познавательных действий (ревизия, сокращение и расширение) для множества убеждений, недостаточен для выражения всех типов изменения, порождаемых различными причинами [14].

Изменение знаний и мнений. Субъект оперирует как знаниями (knowledge), так и мнениями (beliefs). Различие, принимаемое в рамках DEL [19]:

- высказывание считается знанием, если субъект рассматривает его как хорошо устоявшуюся, подтвержденную истину и менее всего склонен от него отказываться;

- убеждением будет считаться скорее то высказывание, которое может быть изменено в результате поступления новой информации, обновления.

Такие определения не предполагают глубокий философский анализ понятий «знание» и «мнение», но скорее, рассматривают их как 
сценарии деятельности субъекта. Указанное различие приводит к разграничению поступающей информации, в зависимости от ее влияния на знания и убеждения субъектов [13].

- Сильные факты (hard facts) - информация, которая изменяет знания и/или убеждения субъектов.

- Слабые факты (soft facts) - информация, изменяющая только убеждения субъектов.

Логический базис. Основа, используемая в методологии DEL, ее логический базис - это DDL K. Сегерберга, расширенная и полная [9], часто называемые нормальной эпистемической логикой. Несмотря на незначительные модификации обозначений, суть базисной логики остается та же. Дальнейшие расширения эпистемических логик исходят из доказанных свойств системы аксиом DDL [10].

Динамизация логики. Методология DEL представляет собой способ динамизации статической логики. Основа - К минимальная логика убеждений. Расширяя базовую статичную логику путем явного определения некоторого действия, можно получить динамизированную логику. Примечательно, что экспликация любого действия в базовой логике осуществляется с помощью ряда редукционных законов (аксиом). Таким образом, любое действие может быть определено в пропозициональном языке и соответственно полученное расширение также будет разрешимым, как и базовая логика [15].

Методология DEL может быть описана таким алгоритмом:

1. Выбор базовой статической логики убеждений и соответствующей модели, позволяющей описывать информационные состояния группы субъектов.

2. Анализ информационных событий - триггеров. Установление их степени влияния на информационное состояние группы, исследование характера соответствующих изменений.

3. Описание события как динамического расширения базовой логики.

4. Описание информационного состояния группы после появления события в пропозициональном языке.

5. Введение аксиом редукции. 
Полученная двухуровневая логика содержит статический базис и динамическое расширение. На уровне базиса существует полная система аксиом для любой выбранной модели. На уровне расширения множество редукционных аксиом, которые сводят динамические действия к пропозициональным выражениям. Таким образом, если разрешим базис, то разрешима и вся логика. Примерами такой динамизации логики способом DEL могут быть логика публичных залялений $P A L$ [13] и построенная на ее основе логика условных убеждений [16].

\section{AGM vs DDL?}

Два основных направления - AGM и динамическая логика DDL, которые определяют концепции исследований пересмотра знаний, имеют ряд существенных различий, но и во многом сходны. Кроме того, существует разветвление и в рамках самих подходов. Оба направления исследуют динамику изменения знаний и убеждений, при этом используя практически универсальный категориальный аппарат и общие исходные принципы. Прежде всего, следует обратить внимание на то, что AGM и DDL это не просто некоторые теории или конкурирующие подходы в области эпистемологических исследований. Скоpeе, это две методологии исследования динамики знаний и убеждений. Основными их отличиями есть выразительная мощность языка, собственно синтаксис, и направленность исследований. AGM модели более общи и создают в основном концепцию явления в целом. DDL модели более выразительны, тщательно проработаны и конкретизированы. AGM подход предполагает более углубленное изучение основных познавательных действий и их свойств. Логики DDL синтаксиса стремятся охватить более широкую предметную область. Более детальное рассмотрение основных принципов обоих подходов дает возможность вьделить две группы характеристик. В первую группу можно отнести свойства, общце для обоих подходов. Ко второй группе отнесем те свойства, в которых DDL и AGM дополняют друг друга. Рассмотрим некоторые характеристики, общие для AGM и DDL.

Исходным принципом для обоих подходов неизменно остается сентенциональность - представ.ление любого убеждения в виде высказывания. Убеждения субъекта представлены в виде множества убеждений, непротиворечивого и замкнутого относительно логического следования. Базовый синтаксис включает в себя пропозициональную логику, которую по необходимости расширяют знаками исследуемых 
операций, термообразующими операторами, модальными операторами.

Системы, разработанные в рамках концепций AGM и DDL, направлены на обработку информационного входа и не содержат переменных времени. Следует отметить, что в рамках динамической логики механизм обработки внешнего входа максимально обобщен, - в качестве побудителя к изменениям может быть рассмотрена и информация, полученная не только извне, но и в результате внутренней активности субъекта.

AGM стратегия базируется на постулатах сокращения. Основные C1-C6 и дополнительные C7-C8 [2]. Постулаты выражают требования, которым должна подчиняться операция сокращения, как наиболее сложно определимое познавательное действие. Эти же ограничения познавательных операций использованы и в динамических логиках на основе DDL. B аксиоматических системах постулаты сокращения отражены в основных аксиомах. При содержательном обосновании DDL систем опять же используются критерии рациональности, разработанные AGM.

Первичные познавательные действия аналогичны в рамках обоих подходов - это расширение и сокращение. Соответственно, обе системы рассматривают ряд вторичных операций, начиная с ревизии (пересмотра) убеждений. Кроме ревизии убеждений рассматриваются и другие познавательные действия, например, консолидация для баз убеждений. Как независимое познавательное действие рассматривается также обновление (update). Стратегия DDL также поддерживает возможность рассмотрения независимых познавательных операций самого различного характера (публичне заявление, лексикографический andeйm и т. п. (см., напр.,: [16], [17]), невыразимых в терминах первичных познавательных действий (сокращения и расширения).

Оба подхода, и AGM, и DDL используют язык, который не дает возможности синтаксически реализовать статус убеждения в теории. В действительности субъект оперирует высказываниями различной степени убежденности и это значительной мерой влияет на процесс изменения убеждений. Упорядочивание, ранжирование, сортировка убеждений в обоих подходах осуществляется с помощью введения различных отношений порядка. В AGM основные исследования свойств отношения порядка на множествах убеждений осуществлены Макинсоном и др. - это так называемая ациклическая иерархия [3]. Отношения порядка, используемые в DDL, основываются на исследованиях эпистемического укоренения [2], рассматриваемых в AGM, и системе 
рангов В. Шпона [12].

B качестве основы для семантических исследований и AGM и DDL используют чаще всего принципы семантики возможных миров C. Крипке, семантики контрфактических высказываний и условных высказываний Д. Льюиса. В DDL активно используются расширения этих систем [4]. Линдстром и Рабинович рассматривают эллиптическое расширение сферической модели А. Грува, классической для AGM. C помошью такого расширения авторы наглядно реализуют индетерминированность ревизии. Еллиптическое расширение Линдстрома и $\mathrm{Pa-}$ биновича предполагает систему сфер Грува как частный случай для детерминированной ревизии [7].

Несмотря на некоторую принципиальную схожесть подходов AGM и DDL, между ними существует ряд существенных отличий. Обратим внимание на некоторые из них.

B AGM нет четко определенного различия между знанияяи субъекта и мнения.ми субъъкта. Наличие некоторого высказывания $\alpha$ в теории преимущественно означает, что некто убежден в $\alpha$, реже-что некто знает $\alpha$. DDL и логики, использующие синтаксис DDL, рассматривают два модальных оператора В (субъект убежден) и К (субъект знает). В первичной системе DDL, разработанной Кристером Сегербергом [10], по умолчанию использован оператор В-убежденности. Более поздние логики на основе DDL, все чапе явно разграничивают мнение и знание, используя обе модальности. Такое разграничение дает возможность исследовать так называемые «слабые» и «сильные» изменения, изменяющие убеждения и знания субъекта [13].

B отличие от AGM динамические логики преимущественно мультисубъектны. В AGM исследование операторов изменения убеждений происходит без явного указания носителя знаний. Неиндексируемая теория К, как множество убеждений некоторого идеального субъекта, вполне достаточна для определения нужных доксастических операций и дальнейшего их анализа. Логики DDL синтаксиса часто используют индексацию, которая призвана отражать мультисубъективность изменения убеждений. В наиболее общем виде эпистемические модальности индексируются подстрочным указанием субъекта (-ов). То есть, запись $K_{i} \alpha$ предполагает, что «субъект $i$ знает $\alpha »$. Также возможно оперирование групповыми, общими знаниями (common beliefs, common knowledges $)-C_{G} \alpha-$ «рруппа G знает, что $\alpha »[17]$.

Функциональное представление AGM операторов изменения убеждений предполагает однозначное определение результата выполнения той или иной операции изменения теории. Поэтому в теориях AGM 
синтаксиса не введены никакие дополнительные операторы, устанавливающие однозначность или вариативность результата изменения. В отличие от однозначности AGM, теории, в основе которых лежит концепция DDL, активно используют следующие и подобные операторы:

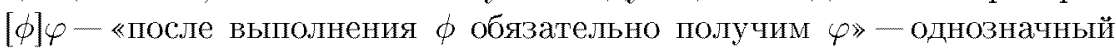
результат, а также дуальный оператор <>, указывающий на возможжную неоднозначность результата. Таким образом, явно указана индетерминированность доксастических операций. В этом случае между исходным множеством убеждений и измененным функциональная зависимость не может быть установлена.

Методология построения динамических логик предполагает создание аксиоматической системы, рассмотрение ее формальных свойств, что и дает право рассматривать этот подход как способ создания логики. Аксиомы таких логик часто выражагот необходимые постулаты AGM [10]. «Статичная», как ее часто называют, модель AGM развивается в функциональном ключе. Принимая за точку отсчета множество постулатов, исследователи рассматривают ключевые свойства и взаимосвязи первичных операторов, способы их реализации, возможности применения.

Для методологии AGM характерно «горизонтальное» развитие. Ряд способов рассмотрения познавательных операторов подразумевает равноценные подходы к описанию познавательного действия (преимущественно сокращение). Завершающим этапом построения определенного вида познавательного действия становится формулировка и доказательство репрезентационной теоремы. С ее помощью устанавливается связь между разработанным подходом и ранее известными способами рассмотрения операторов. В целом, основные концепции AGM попарно әквивалентны. Это установлено посредством ряда репрезентационных теорем, например, см.: [3].

Для логик DDL синтаксиса характерно «вертикальное» развитие экспансия. На основе аксиоматической системы, которая обладает уже доказанными свойствами и разработанным синтаксисом, строится новая аксиоматическая система - расширение основной. Основным достижением DDL подобных логик, стало доказательство полноты аксиоматической системы Full DDL Кристером Сегербергом [9]. Full DDLполная система динамической доксастической логики, разработанная Линдстромом и Рабиновичем [8]. Она дает возможность оперировать формулами вида $[O \alpha] B \beta$, где $\mathrm{O}-$ некоторое доксастическое действие, B - оператор убежденности, $\alpha$ и $\beta$ могут быть любыми высказываниями, как о внешнем мире, так и о эпистемическом состоянии самого 
субъекта. Фактически, это дает возможность снять ограничение предметной области $\alpha$ и $\beta$, которые представляют собой эпистемический вход и результат изменения. Субъект Full DDL может оперировать информацией любого рода-от используемой и AGM информации о внешнем мире, до своих внутренних потребностей и желаний. Естественно, это значительно расширяет сферу применения DDL, но и соответственно усложняет ее структуру.

При построении очередной динамической логики, в ее основу ложится базовый синтаксис DDL (иногда в измененном виде). В конечной системе обычно приводится ряд редукционных аксиом, позволяющих выразить вводимые исследователем дополнительные обозначения в базисном языке. Завершаюшим этапом построения такой аксиоматической системы становится рассмотрение ее формальных свойств и доказательство соответствующих теорем на основе уже доказанных теорем о свойствах базисной логики DDL.

Исследование динамики изменения убеждений в рамках AGM подхода реализуется с помощью анализа механизмов познавательных действий и однозначного определения познавательньх операций. Логики DDL синтаксиса динамизируются с помощью методологии DEL: paсширения статичной базовой логики DDL с помощью динамических операторов - действий, выразимьх в базовом языке. В качестве действий DEL использует публичное заявление, различные виды обновлений и др. [19].

Итак, AGM vs DDL? Не совсем так. Нельзя говорить о каких-то неразрешимых противоречиях между этими двумя подходами. Также нельзя и сказать что одна система удобнее, выразительней, полезней другой. В некоторых случаях да, такое замечание будет уместным, но лишь в первом приближении. Фактически AGM и DDL это два уровня развития доксастических и эпистемических исследований. Если AGM выполняет основную работу на описательном метауровне [5, с. 39], который «задает тон» предметным изысканиям, то DDL - логики реализуют и расширяют предметную область применения доксастических исследований. В рамках AGM осуществляются формальные исследования идеализированных объектов с целью установления общих свойств, построения общей концепции и методологии. DDL синтаксис дает возможность реализовать доксастический подход для анализа динамических процессов в различных областях человеческой деятельности - системы принятия решений, компьютерные игры, формирование общественного мнения и др. [14]. 


\section{5 Литература}

[1] Alchourron C.E., Gärdenfors P., Makinson D. On the Logic of Theory Change: Partial Meet Contraction and Revision Functions. Journal of Symbolic Logic, 50: 510 - 530, 1985.

[2] Gärdenfors P., Rott H. Belief revision. In Handbook of Logic in Artificial Intelligence and Logic Programming, volume IV, chapter 4.2, 1995 .

[3] Gärdenfors P. Knowledge in Flux. Modeling the Dynamics of Epistemic States. Cambridge, Massachusetts: The MIT Press, 1988.

[4] Girard P. From onions to broccoli: generalizing Lewis' counterfactual logic. In Andreas Herzig and Hans van Ditmarsch, editors, Belief revision and dynamic logic, Journal of applied non-classical logic. HermesLavoisier, 2007.

[5] Hansson S.O. Ten Philosophical Problems in Belief Revision. Journal of Logic and Computation, 13(1):37-49, 2003.

[6] Hintikka $J$. Knowledge and belief: an introduction to the logic of the two notions. Ithaca, N. Y.: Cornell University Press, 1962.

[7] Lindstrom S., Rabinowicz W. Epistemic entrenchment with incomparabilities and relational belief revision, pp. 93-126 in The Logic of Theory Change, edited by A. Fuhrmann and M. Morreau, Springer-Verlag, Berlin, 1991.

[8] Lindstrom S., Rabinowicz W. Extending dynamic doxastic logic: accommodating iterated beliefs and Ramsey conditionals within DDL. In For good measure, edited by Lars Lindahl, Paul Needham \& Rysiek Sliwinski, pp. 126-153. Uppsala Philosophical Studies, vol. 46, 1997.

[9] Segerberg K. A Completeness Proof In Full DDL Logic and Logical Philosophy, Volume 9, pp.77-90, 2001.

[10] Segerberg $K$. Belief revision from the point of view of doxastic logic. Bulletin of the IGPL, vol. 3, 1995.

[11] Segerberg K. Further questions about hypertheories. Uppsala Philosophical Studies, vol. 45. Uppsala, 1997. 
[12] Spohn W. Ordinal Conditional Functions: A Dynamic Theory of Epistemic States, Harper W. L. et al.., Ed., Causation in Decision, Belief Change and Statistics II, pp. 105-134, Kluwer, Dordrecht, 1988.

[13] Van Benthem J. Dynamic logic for belief change. In Andreas Herzig and Hans van Ditmarsch, editors, Belief revision and dynamic logic, Journal of applied non-classical logic. Hermes-Lavoisier, 2007.

[14] Van Benthem J. Open Problems in Logical Dynamics, In T.Rozhkovskaya, ed., Mathematical Problems from Applied Logics, Springer, New York, 2006.

[15] Van Benthem J., Kooi B. Reduction axioms for epistemic actions. In R. Schmidt, I. Pratt-Hartmann, M. Reynolds, and H. Wansing, editors, Preliminary Proceedings of AiML-2004, pp. 197-211, 2004.

[16] Van Benthem J., Lio F. The dynamics of preference upgrade. Journal of Applied Non-Classical Logics, 2007.

[17] Van Ditmarsch H., Verbrugge R. Common Knowledge. In: Jan van Eijck and Rineke Verbrugge (editors), Discourses on Games, Action, and Social Software. Texts in Logic and Games, Amsterdam University Press, 2008.

[18] Van Ditmarsch H. Comments to 'Logics of Public Communications'. Synthese (Knowledge, Rationality \& Action) 158(2): 181-187, 2007.

[19] Van Ditmarsch H., Kooi B., Van Der Hoek W. Dynamic Epistemic Logic. Synthese Library, 2007. 